\title{
Los ciclos de manejo, una herramienta útil si es dinámica para evaluar el consumo de combustible y las emisiones contaminantes del auto transporte
}

\author{
R. González-Oropeza \\ Laboratorio de Control de Emisiones, Facultad de Ingeniería \\ Universidad Nacional Autónoma de México \\ E-mail:rog1950@servidor.unam.mx
}

(recibido: agosto de 2004; aceptado: febrero de 2005)

\section{Resumen}

Una revisión de la literatura especializada sobre los ciclos de manejo que se han desarrollado y que se siguen desarrollando en distintos países, permite hacer algunas reflexiones sobre el tema, además de comentar sobre las actividades de experimentación que se han llevado a cabo en el Laboratorio de Control de Emisiones de la Facultad de Ingeniería. Los investigadores de distintos países que trabajan sobre este tema, han reportado cambios en los patrones de conducción, el Federal Test Procedure, que fue de los primeros ciclos que se desarrollaron (inició a mitad de los 60's) ha sufrido cambios desde muchos, años atrás para estar actualizado como se menciona en EPA (1998). De igual forma, algunos países de la Unidad Europea han desarrollado diferentes ciclos de manejo, y al compararlos con el EURO93, se comenta que debe actualizarse, ya que no satisface los requisitos para reproducir las condiciones de conducción reales, principalmente en zonas urbanas (André M. Robert et al., 2001)

Hoy en día, no sólo son preocupantes las emisiones que afectan directamente a la salud, sino también el uso inmoderado de los combustibles, ya que además de consumirse productos no renovables, se generan grandes volúmenes de uno de los gases de efecto invernadero, el $\mathrm{CO}_{2}$. Debería existir un compromiso real de todos los países del orbe, principalmente de aquellos altamente industrializados para que reduzcan la generación de $\mathrm{CO}_{2}$, y como uno de los sectores que consumen grandes volúmenes de energéticos es el del auto transporte, es inquietante saber qué se está haciendo al respecto. En el presente artículo se mencionan algunos puntos de vista acerca de estas actividades.

Descriptores: Ciclos de manejo mexicanos, evaluación del consumo de combustible y de las emisiones contaminantes en el transporte por carretera, un panorama mundial de los ciclos de manejo.

\footnotetext{
Abstract

A review of the specialized literature over driving cycles which have been developed in many cities and countries, allow to make some reflections over this topic, and to comment about the experimentation activities (on the roads, motorways, in the laboratory and by computational models). The researchers that work in this field have reported substantial changes in the driving patterns. Federal Test Procedure was one of the first cycles to be developed (it was known in the decade of the 60's) and it has suffered changes from many years ago in order to be modernized EPA (1998). In the same way, many countries of the European Comunity have developed different driving cycles and when these have been compared with
} 
Los ciclos de manejo, una herramienta útil si es dinámica para evaluar el consumo de combustible ...

\begin{abstract}
EURO93, it was commented that the actual cycle should be modernized because it doesn't reproduce the real world conditions, mainly in urban areas (André M. Robert et al., 2001).

Nowadays not only should we be concerned by the emissions that directly affect health, but also the exaggerated fuel consumption. In addition to wasting non-renewable products, it generates large volumes of one of the greenhouse gases, $\mathrm{CO}_{2}$.

A commitment exist in all countries, mainly those which are highly industrialized to reduce the generation of $\mathrm{CO}_{2}$, and as Road Transport is one of the sectors that consumes largest volumes of energy it's interesting to know what is being done in this respect. In this paper some points of view are mentioned concerning the researchers (from various countries) about the activities that Road Transportation develop to decrease fuel consumption and emissions, and therefore comply with the goals of Kyoto.
\end{abstract}

Keywords: Mexican driving cycles, assessment fuel consumption and polluting emissions of road transportation, a world scope of the driving cycles.

\section{Introducción}

Un ciclo de manejo es un perfil de velocidades trazado en un plano velocidad-tiempo, que representa una forma típica de conducir en una ciudad o autopista, tomando en cuenta la tecnología del vehículo, las características del tráfico, de las carreteras, características climáticas y geográficas (altitud, entre las más importantes) y también características de los mismos conductores.

Estos ciclos de manejo tienen una gran importancia, entre otros fines, para planear adecuadamente el desarrollo de alguna ciudad, en el desarrollo de tecnología para los nuevos automóviles, en la validación de modelos que predicen el comportamiento de los vehículos en la vía pública y en los inventarios de emisiones contaminantes en las grandes urbes, los cuales a su vez, permiten establecer estrategias para controlar el equilibrio ecológico del lugar, ciudad o región; entendiéndose éste como "la relación de interdependencia entre los elementos que conforman el ambiente que hace posible la existencia, transformación y desarrollo del hombre y demás seres vivos (SEMARNAT).

Las condiciones de tráfico cambian permanentemente debido a varios factores, como son: el crecimiento de la población, tecnología de los vehículos, cambios en el transporte público, modificaciones en la red de carreteras, nuevas legislaciones para mantener el equilibrio ecológico, etc. Esto hace que las formas de conducir sean dinámicas, o dicho en otras palabras, que vayan cambiando conforme exista una mayor demanda del transporte en el contexto descrito anteriormente.
Si no se atiende esta dinámica de cambio, es decir, si no se actualizan los ciclos de manejo, éstos dejan de ser una herramienta confiable para estimar los inventarios de emisiones, certificación u homologación de vehículos, medición del consumo de combustible, etc.

En cuanto a lo que se ha hecho en México, el grupo de trabajo del Laboratorio de Control de Emisiones (LCE), ha desarrollado 5 ciclos de manejo que se describen someramente en este trabajo y que corresponden a las zonas Noroeste, Noreste, Centro, Suroeste y Sureste de la Zona Metropolitana del Valle de México.

\section{Comparaciones de los ciclos desarrollados actualmente con los ciclos vigentes en las legislaciones respectivas}

\section{USA}

La EPA (Environmental Protection Agency, 1998), llevó a cabo una revisión del ciclo de manejo federal (Federal Test Procedure) contratando para ello a Radian Corporation y a Sierra Research Inc., quienes realizaron sus muestreos en las ciudades de Baltimore y Spokane, así, los datos aquí expuestos han sido consultados del reporte preliminar correspondiente presentado en marzo 27 de 1998. En el documento se menciona que el FTP es más conservador que los datos obtenidos en Baltimore, de esta manera la tabla 1 muestra las diferencias entre velocidad, aceleración y potencia demandada, respectivamente. 


\section{R. González-Oropeza}

Tabla 1. Resumen estadistico entre los datos del FTP y Baltimore

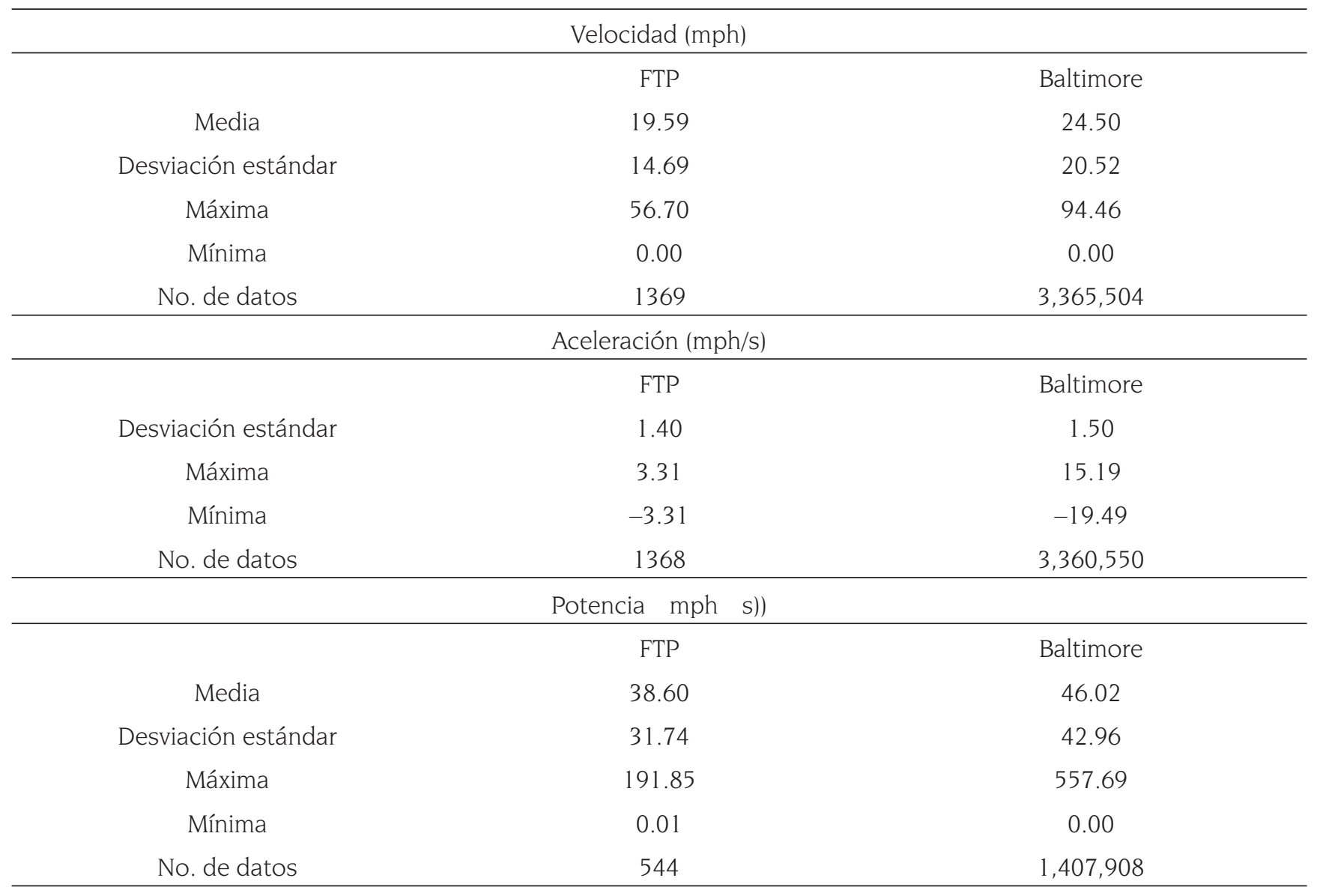

El FTP no contiene velocidades por encima de $56.7 \mathrm{mph}$, y la aceleración, ya sea negativa o positiva, no excede los valores de $3.3 \mathrm{mph} / \mathrm{s}$. En cambio, en el caso de Baltimore, el $8.5 \%$ de las velocidades está por encima de $56.7 \mathrm{mph}$, y con respecto a la aceleración, el $2.5 \%$ excede al valor de $3.3 \mathrm{mph} / \mathrm{s}$, mientras que $3.5 \%$ está por debajo del valor $-3.3 \mathrm{mph} / \mathrm{s}$.

Respecto al método de recolección de datos, los vehículos fueron instrumentados con sistemas de adquisición de datos (datalogger) para registrar:

1. La velocidad del vehículo,

2. Régimen de giro (rpm) y

3. Presión de vacío en el múltiple de admisión;

sin embargo, los fabricantes de vehículos solicitaron participar en este estudio y se anexó un programa al adquisidor de datos, así como los sensores respectivos para registrar:
4. La temperatura del refrigerante,

5. La posición de la válvula de mariposa y

6. El dosado (relación combustible/aire) aguas abajo del catalizador,

así que unos vehículos registraron 3 parámetros y otros 6. Cerca de 730 vehículos fueron instrumentados en Baltimore y Spokane y los sistemas registraron más de 6 millones de segundos del comportamiento de manejo en las 2 ciudades.

Para tener la certeza de que los datos registrados fueran confiables, se utilizó también la medición de velocidad a distancia y además, los valores obtenidos se compararon con los logrados por un coche "caza" (vehículo que sigue al que realiza el muestreo y que dispone de instrumentos para registrar la velocidad de éste) usado en Los Angeles, ya que el estudio patrocinado por el CARB en Los Angeles se llevó a cabo simultáneamente al estudio patrocinado por la EPA en Spokane; de dicha comparación se concluyó que los datos son perfectamente compatibles. 
Los ciclos de manejo, una herramienta útil si es dinámica para evaluar el consumo de combustible ...

Tabla 2. Comparación entre los datos obtenidos con un coche "caza" y también mediante la instrumentación de vehículos

\begin{tabular}{|c|c|c|c|c|}
\hline \multirow{2}{*}{$\begin{array}{l}\text { Mediciones del } \\
\text { comportamiento de manejo }\end{array}$} & \multicolumn{2}{|c|}{ Baltimore } & \multicolumn{2}{|c|}{ Spokane } \\
\hline & Carro Caza & $\begin{array}{c}\text { Vehículo } \\
\text { Instrumentado }\end{array}$ & Carro Caza & $\begin{array}{l}\text { Vehículo } \\
\text { Instrumentado }\end{array}$ \\
\hline \multicolumn{5}{|l|}{ Velocidad (mph) } \\
\hline Promedio & 30.70 & 24.50 & 29.80 & 23.24 \\
\hline Máximo & 79.50 & 94.46 & 83.20 & 77.55 \\
\hline Desviación estándar & 21.00 & 20.52 & 19.50 & 1771 \\
\hline No. de datos & 191,119 & $3,365,504$ & 175,137 & $2,081,199$ \\
\hline \multicolumn{5}{|l|}{ Aceleración (mph/s) } \\
\hline Mínima & -20.80 & -19.49 & -11.30 & -15.46 \\
\hline Máxima & 8.13 & 15.19 & 7.79 & 15.95 \\
\hline Desviación estándar & 1.39 & 1.50 & 1.42 & 1.46 \\
\hline \multicolumn{5}{|l|}{ Potencia mph s)) } \\
\hline Promedio & 22.2 & 19.28 & 20.00 & 17.01 \\
\hline Máxima & 577.30 & 557.69 & 403.00 & 672.28 \\
\hline Long. de viaje prom. (mi) & 7.48 & 4.89 & 5.83 & 3.56 \\
\hline Tiempo prom. de viaje (min) & 14.61 & 12.03 & 11.72 & 9.18 \\
\hline
\end{tabular}

Desde un principio, la EPA conocía la habilidad del carro "caza" para registrar los datos de conducción de forma agresiva, que también fue parte de los objetivos del estudio, sólo una pequeña parte de vehículos que fueron conducidos de esta forma escaparon a la medición de su velocidad porque cambiaban de carril (línea) o porque era inseguro para el carro "caza" (Tabla 2).

Es bien sabido que existen aceleraciones que no están representadas por el FTP; sin embargo, podría ser contra productivo modificar el FTP a menos que se cumplieran dos condiciones: la primera, que la forma de conducir no estuviera representada apropiadamente por el FTP y que esta situación provocara emisiones contaminantes en una cantidad significativa, si eso no ocurría así, entonces modificar el FTP podría significar excesivos costos y disturbios y sólo lograr pequeños beneficios o ninguno en la calidad del aire; y la segunda, cualquier modificación al FTP podría redundar en mejorar el diseño de los vehículos, por lo tanto, controlar de mejor forma las emisiones contaminantes.

El FTP es más grande en duración y distancia que los viajes medidos en Baltimore, aunque el FTP es muy parecido a los viajes en Baltimore por la mañana.

Otro aspecto más de comparación es el período de reposo, el FTP incluye 2, uno de 10 minutos y otro que dura toda la noche, en cambio, casi el $40 \%$ del tiempo de reposo en Baltimore fue entre 10 minutos y 2 horas, que es el período más crítico para el enfriamiento del catalizador. Los datos demuestran que el FTP exagera en la proporción de arranques con el catalizador "caliente" y también en la proporción de arranques con el motor frío. Implícitamente asume que el 57\% de todos los arranques se llevan a cabo con el catalizador caliente, mientras que los datos actuales indican que sólo el 30\%. También asume que el 43\% de todos los arranques son con el motor frío, mientras que los datos actuales sugieren que menos del 25\% ocurren con la temperatura del motor igual a la temperatura ambiente $\pm 25^{\circ} \mathrm{F}$.

Desde el punto de vista global de un inventario de emisiones, estos dos últimos factores se pueden compensar mutuamente, la proporción del catalizador caliente con la proporción del motor frío; sin embargo, los períodos de reposo de 10 minutos y de toda la noche, no ofrece incentivos a 


\section{R. González-Oropeza}

los fabricantes para demorar el enfriamiento del catalizador. Esto es, si el descanso es de $10 \mathrm{mi}-$ nutos no necesita retardarse el enfriamiento, la temperatura del catalizador está por encima de la que tendría en un arranque en frío, y si dura toda la noche, no se puede mantener esa temperatura por tanto tiempo. Por lo tanto, se piensa que para no perder la oportunidad de mantener por más tiempo una temperatura adecuada del catalizador (por ejemplo con un aislante apropiado), podría incluirse en el FTP un período de reposo intermedio.

Finalmente, se comenta un aspecto por demás interesante, el cual debe tenerse en cuenta al proponer un ciclo de manejo, "las pendientes del camino".

La Agencia de Protección al Ambiente (EPA), mostró en 1980, una distribución de las pendientes de las carreteras en USA, donde se puede apreciar que el $30 \%$ de los viajes se realizan por carreteras con más del $2 \%$ de pendiente, el 35\% entre 0.5 y $2 \%$ de pendiente y el resto con menos de $0.5 \%$. En contraposición con estos datos, el trazo del FTP se transcribe al equipo del dinamómetro de chasis, asumiendo que se trata de una superficie plana o llana, ya que fue desarrollado sin tomar en cuenta dichas pendientes (Esteves et al ., 2001). De esta forma, las cargas y aceleraciones causadas por las pendientes y rampas no son adecuadamente representadas. La comparación del FTP con las condiciones de manejo vigentes es muy simple, el FTP asume caminos llanos y el mundo real no es así.

\section{La Unión Europea}

Un gran número de países pertenecientes a la Unión Europea, principalmente en la última década, han desarrollado muchas y muy variadas actividades tendientes a proponer ciclos de manejo más apropiados a su realidad, que reproduzcan las condiciones de manejo de la vida actual, tanto en la ciudad como en la autopista.

El ciclo de manejo europeo no contiene muchas aceleraciones o etapas transitorias. El ciclo repite la parte urbana 4 veces, y la mayor aceleración que registra es de velocidad cero hasta $50 \mathrm{kph}$ en 28 segundos, dicho ciclo es, por lo tanto, escasamente representativo de las condiciones de manejo normales (Per Kagenson, 1998).
Muestreos con vehículos instrumentados en Italia, Francia, Gran Bretaña y Alemania (André M.) permitieron comparar los datos obtenidos con algunos ciclos ya existentes (Europeo, Japonés y Americano), coincidiendo algunas velocidades promedio en las zonas urbanas: FTP72, 11-modos Japonés, 10-modos Japonés y el Europeo, pero en este último la proporción de velocidades estables es muy alta y la frecuencia en los cambios aceleración-desaceleración es el peor parámetro descrito. El promedio de los valores de aceleración son sustancialmente más bajos que en la realidad. Algunos investigadores comentan que con algunos ajustes menores, el FTP72 describe razonablemente bien las condiciones de tráfico promedio en las ciudades Europeas, mientras que el ciclo Europeo es totalmente inadecuado.

Existe otro parámetro de suma importancia que, paralelamente al medir los niveles de las emisiones contaminantes, debe conocerse para construir los inventarios de emisiones, el Consumo de Combustible, el cual puede estar incluido al desarrollar ciclos de manejo. Los acuerdos de Kyoto de 1997, son un reflejo de la preocupación mundial en la producción de $\mathrm{CO}_{2}$ que es uno de los gases que tienen mayor importancia en el "efecto invernadero" y que tiene una relación muy estrecha con el consumo de combustible. Al respecto, ya han aparecido trabajos relacionados con este tema.

La tabla 3, aunque con datos de hace 15 años aproximadamente, muestra las diferencias entre los valores de las pruebas realizadas en laboratorios y los obtenidos a partir del uso cotidiano de los vehículos. La literatura menciona los factores que afectan el consumo de combustible, pudiéndose agrupar en tres tipos:

$1^{\circ}$ Inherentes al conductor (patrón de manejo, que incluso tiene influencia en la eficiencia del motor),

$2^{\circ}$ Factores inherentes al vehículo, también frecuentemente llamada "carga de camino" que se refiere a la resistencia al rodamiento (peso, velocidad y tipo de neumáticos), resistencia al arrastre (geometría del vehículo) y

$3^{\circ}$ La tecnología del motor, tipo de combustible (gasolina, diesel, GLP, mezcla), desplazamiento o 
Los ciclos de manejo, una herramienta útil si es dinámica para evaluar el consumo de combustible ...

cilindrada, relación de compresión, tiempo de inyección/ignición, sistema de distribución, válvulas por cilindro, presencia de auxiliares como un turbocompresor o intercooler y el tipo de motor (encendido por chispa y encendido por compresión).

En 1998, la Comisión Europea y la Asociación de Fabricantes de Carros Europeos (ACEA, European Car Manufacturers Association) acordaron que el promedio de carros vendidos en 2008 no debería emitir más de $140 \mathrm{~g}$ de $\mathrm{CO}_{2}$ por $\mathrm{km}$ recorrido, dicho valor obtenido con el nuevo ciclo europeo de pruebas para consumo específico de combustible, el Eurotest de la Directiva 93/1 16/EC, esto significa una mejora del $25 \%$ del consumo específico de combustible, respecto al que se tenía en 1997. Otra parte del acuerdo consistió en la introducción, alrededor del año 2000, de carros de pasajeros emitiendo menos de $120 \mathrm{~g}$ de $\mathrm{CO}_{2} / \mathrm{Km}$. En este sentido, la política Holandesa anunció sus planes para reducir la emisión de gases de efecto invernadero, siendo uno de los más importantes el $\mathrm{CO}_{2}$. "Los vehículos se clasificarían en grupos de tamaño comparable entre ellos mismos y el promedio del consumo específico de estos grupos será calculado todos los años, de manera que si un carro nuevo tiene un consumo menor que dicho promedio se le tasará con un impuesto menor al normal, en cambio, si su consumo específico de combustible es mayor que el promedio, pagará mayor impuesto".

A pesar de esta política, la medición del consumo específico de combustible mediante el nuevo ciclo de pruebas europeo, es en general, más bajo que el consumo real y las razones son las siguientes:

- La aceleración del vehículo y la velocidad del motor (rpm) en el Eurotest son mas bajas que en la realidad (el promedio de aceleración en el Eurotest es de $0.5 \mathrm{~m} / \mathrm{s}^{2}$, mientras que el promedio en ciclos de prueba más cercanos a la realidad es de $0.7 \mathrm{~m} / \mathrm{s}^{2}$ )

- Los fabricantes preparan a sus vehículos para el Eurotest, a fin de lograr el menor consumo de combustible posible. Incluso se cree que esta diferencia se acentúe con las novedades tecnológicas, como los motores de encendido por chispa que emplean una inyección directa estratificada, y por tanto, tienen un consumo menor.
- El Eurotest se realiza con neumáticos estándar (pequeños) y sin auxiliares (aire acondicionado, por ejemplo) mientras que muchos propietarios de automóviles cambian los neumáticos por otros más anchos y utilizan muchos de los auxiliares disponibles.

Una comparación realizada también en Holanda, entre el Eurotest y la forma real de manejo (utilizando 120 vehículos) mostró que el ciclo europeo estima un $10 \%$ menos del consumo de combustible que mediante la forma habitual de manejo. Los carros alemanes estiman esta diferencia en 17\%; sin embargo, debe tenerse en cuenta que en Alemania no existe límite de velocidad en la mayoría de sus autopistas (Tabla 3).

La EPA en Estados Unidos desarrolló un ciclo de manejo para determinar la economía del combustible de vehículos ligeros (Svensk Bilproving Motorcenter, 1997), denominado HWFET (High Way Fuel Economy Test). Es un programa para dinamómetro de chasis, con una duración de $765 \mathrm{~s}$, una distancia total recorrida de $16.45 \mathrm{~km}$ y velocidad promedio de $77.7 \mathrm{kph}$ (Figural).

En otra línea de investigación, algunos grupos intentan desarrollar el modelo más completo para evaluar las emisiones y el consumo de combustible, en función de los patrones de manejo que se generan por el propio modelo (Ericsson, 2001).

Existen hoy en día modelos que pueden reproducir casi a la perfección patrones de manejo, valores de las emisiones y el consumo de combustible; no obstante, siempre existe la desventaja de que los ciclos generados por dichos modelos no incluyen algunas variaciones del comportamiento real de las variables, lo cual puede causar erráticos resultados y conclusiones. Estos ciclos de manejo corren el riesgo de estar suavizando algunos aspectos en comparación con los datos reales del tráfico. 
DOI: http://dx.doi.org/10.22201/fi.25940732e.2005.06n3.011

R. González-Oropeza

Tabla 3. Diferencia en rendimiento de combustible, pruebas/realidad de varios paises (1/100 km)

\begin{tabular}{|c|c|c|c|c|c|c|}
\hline País & Año & Prueba & Real & $\begin{array}{l}\text { Diferencia } \\
\text { promedio }\end{array}$ & $\%$ dif & Comentarios \\
\hline Canadá a & 1988 & 8.0 & 10.0 & 2.0 & 20 & \multirow{2}{*}{$\begin{array}{l}\text { Rendimiento real de las encuestas a los } \\
\text { conductores. Pruebas de laboratorio. }\end{array}$} \\
\hline Modelo indiv. & 1985 & 8.6 & 10.7 & 2.1 & 19.6 & \\
\hline Francia $^{c}$ & 1988 & 6.5 & 8.4 & 1.9 & 23 & $\begin{array}{l}\text { Viajes diarios comparando, } 1 / 3 \text { ciudad, } \\
1 / 3 \text { autopista, } 1 / 3 \text { carreteras. }\end{array}$ \\
\hline Alemania d & 1987 & 7.7 & 9.8 & 2.1 & 21.4 & Pruebas DIN vs. DIW (real). \\
\hline Suecia e & 1987 & 8.2 & 8.5 & 0.3 & 3.5 & $\begin{array}{l}\text { KOV comparado con lo reportado en las } \\
\text { encuestas. }\end{array}$ \\
\hline USA ${ }^{\mathrm{f}}$ autos & 1985 & 9.7 & 11.9 & 2.2 & 18.5 & $\begin{array}{l}\text { Encuesta RTECS vs. flotas promedio de la } \\
\text { EPA de las pruebas en dinamómetro. }\end{array}$ \\
\hline Camiones & 1985 & 11.6 & 14.5 & 2.29 & 20 & \\
\hline $\mathrm{UK}^{\mathrm{g}}$ & 1989 & 7.2 & 9.3 & 2.1 & 22.6 & $\begin{array}{l}\text { Valor de prueba para el promedio registro } \\
\text { - pesado. }\end{array}$ \\
\hline
\end{tabular}

Fuentes:

a Estadísticas Canadá, 1990

e KOV, 1987

b SOM. Inc 1988: Energía, Minas y Recursos, 1992

${ }^{\mathrm{f}}$ Mintz y col., 1993

c Bosseboeuf, 1988

g Sorrell, 1992

d DIW, 1987

(Lee y Wienke, 1994)

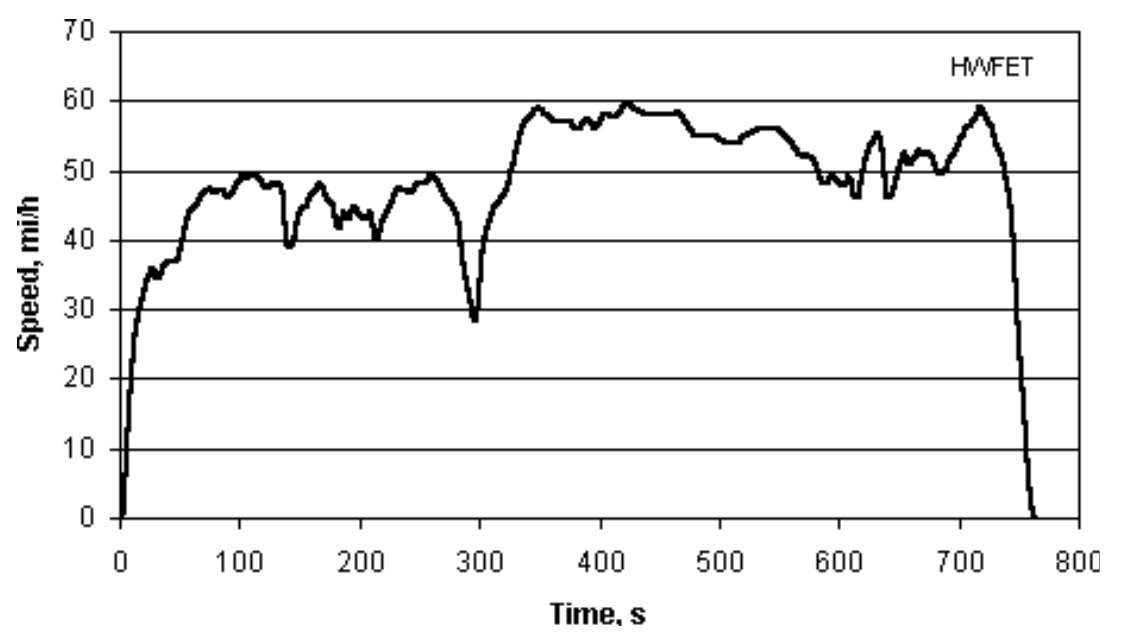

Figura 1. Ciclo para la economía del combustible, EPA

\section{Lo más reciente}

En el contexto que se ha descrito anteriormente, La Comisión Europea ha establecido proyectos de investigación que tienen una gran relevancia como el HyZEM (European Development of Hybrid Vehicle Technology Approaching Efficient Zero Emission Mobility), ARTEMIS (Assessment and
Reliability of Transport Emission Model and Inventory System), y MODEM (MODelling of EMissions and Fuel Consumption in Urban Areas) (Michel, 2001), cuyos principales objetivos son los siguientes:

- Establecer un conjunto de ciclos de manejo que representen la forma de conducir real, 
Los ciclos de manejo, una herramienta útil si es dinámica para evaluar el consumo de combustible ...

para ser usados en el marco de estos proyectos, pero también en las campañas nacionales para medir las emisiones contaminantes, de forma que se asegure la compatibilidad e integración de los resultados en el sistema europeo de inventario de emisiones.

- Asegurar y validar la representatividad de la base de datos y ciclos de manejo a través de la comparación, tomando en cuenta datos complementarios.

- Incluir en 3 tipos de ciclos (urbano, carretera y autopista) la diversidad de condiciones de manejo observadas, y dentro de sub-ciclos, permitir la desagregación de las emisiones de acuerdo a condiciones de manejo más específicas como tráfico congestionado, flujo libre urbano, etc.

Para reunir toda esta información, diferentes países e instituciones han aportado los datos que recolectaron en varias ciudades, carreteras y autopistas europeas durante los pasados 10 años, así que se generaron los ciclos denominados:

- MODEM and MODEM-IM CYCLES, datos de 58 autos privados que se monitorearon en Francia, UK y Alemania y que representan el parque vehicular respectivo. Se completaron un total de 1400 días, 73,000 km, 8,200 viajes y 1,680 horas de manejo. Se tienen ciclos urbanos, urbano lento, urbano de libre flujo, de carretera y de autopista.

- MODEM-HyZEM CYCLES, se anexaron a los datos anteriores, los resultados de monitoreos realizados en Grecia con 20 automóviles privados. Todos los vehículos han sido equipados con sistemas de adquisición de datos y conducidos por sus propios dueños. En conjunto, los datos sumaron 2,000 días, 10,300 viajes, $88,000 \mathrm{~km}$ recorridos y 2,200 horas de manejo. Se obtuvieron 8 ciclos que describen situaciones específicas en el área urbana, en carretera y en autopista.

- ARTEMIS-CYCLES, se usaron las bases de datos de modem-Hyzen, pero ahora se aumentó la información con los datos de Suiza e Italia $(16,600 \mathrm{~km})$. Se validaron los ciclos generados al ser comparados con los últimos datos proporcionados por estos países. Se construyeron 3 ciclos: urbano, carretera y autopista. Se describen 12 situaciones específicas en los subciclos dentro de los 3 ciclos mencionados.

- INRETS-PVU, un muestreo con 39 vehículos de servicio ligero, 12 automóviles de pasajeros, 12 Vans ligeras y 15 Vans de 2 a 3.5 toneladas, fueron utilizados para registrar los datos de 9,728 viajes, $78,500 \mathrm{~km}, 1,100$ días y 2,160 horas de manejo. Se siguió básicamente el mismo método que en los casos anteriores, generándose de 6 a 9 ciclos dependiendo de la categoría del vehículo y describiendo situaciones como: urbana libre, urbana densa, mensajería, carretera y autopista, aplicando carga pesada y ligera al vehículo.

La descripción de la enorme diversidad de condiciones de manejo se afronta con el conjunto de los 12 agrupamientos o clases, que son configuraciones de carreteras y condiciones de tráfico, derivados directamente del análisis factorial y clasificación automática de los datos del modem-Hyzem. Los agrupamientos están afectados de sus pesos respectivos (en km o duración) en la base de datos inicial.

\section{Ciclos de manejo para autobuses}

Las nuevas tecnologías incrementan la vida útil de los vehículos y el número de kilómetros recorridos, que es mayor que el de los carros viejos, así que debería existir una legislación que mantenga ciertos limites de emisiones contaminantes y de consumo de combustible durante la vida útil del vehículo.

Los autobuses son el soporte principal del transporte público en la mayoría de las ciudades, incluso donde existen esquemas de transporte mediante trenes o trenes ligeros; sin embargo, las emisiones contaminantes y el consumo de combustible (emisión de $\mathrm{CO}_{2}$ ) de los autobuses provistos de motor diesel, ha sido un problema controversial. A pesar de las sorprendentes mejoras que se han hecho a estos motores en los últimos años, los gases de escape contienen más de 40 sustancias que son consideradas como tóxicas y unas 15 que son consideradas carcinógenas.

Es ampliamente conocido que la operación de un autobús a bajas velocidades en condiciones de "paro-arranque" (congestionamientos) exacerba 


\section{R. González-Oropeza}

enormemente las emisiones, y para medir adecuadamente dichas emisiones y el consumo de combustible, la Agencia de Protección al Medio Ambiente de Nueva Gales del Sur (New South Wales Environmental Protection Agency, NSW EPA) bajo un contrato separado con el Consejo Nacional de Protección del Medio Ambiente (NEPC) de Australia, desarrollaron un conjunto de ciclos de manejo transitorios que representan las condiciones del "mundo real" (Anyon, 2001).

Estos ciclos de manejo denominados "Composición de Ciclos de Manejo para las Emisiones Urbanas" (Composite Urban Emissions Drive Cycles, CUEDC) fueron sintetizados de un extenso número de datos generados mediante la instrumentación de vehículos en Sidney, en la región metropolitana. Dichos ciclos simulan los patrones de manejo agresivo y las velocidades típicas de las grandes ciudades Australianas.

El trazo velocidad-tiempo del CUEDC es aplicable a los vehículos de categoría NC, de 12.0 a 25.0 toneladas de peso bruto vehicular. Se distinguen 4 perfiles principales: congestionado (328 segundos), manejo en carreteras secundarias (509 segundos), arterias (431 segundos) y autopista (528 segundos).

- El segmento congestionado está caracterizado por bajas velocidades, aceleraciones y frenados frecuentes separados por períodos cortos de tiempo inactivo o en vacío (sin carga, con velocidad cero o muy bajas).

- Manejo en carreteras secundarias, es principalmente por debajo de $60 \mathrm{kph}$, con períodos de descanso que no son frecuentes, indicando un tráfico de flujo libre, glorietas y vueltas en las intersecciones señaladas por los semáforos.

- El manejo en arterias tiene rápidas aceleraciones para alcanzar velocidades más altas, esparcidas con períodos de descanso en los semáforos.

- El manejo en autopista tiene velocidades aun más altas, con pocas interrupciones y períodos más extendidos con velocidad de crucero.

Se ha mostrado claramente que la única forma de evaluar los niveles de emisiones, es aplicarle carga a un vehículo en un dinamómetro de rodillos, el cual simula condiciones reales de manejo. Es importante mencionar lo anterior porque hasta ahora las legislaciones en todos los países aplican pruebas solamente a los motores de los vehículos pesados.

Hasta hace poco tiempo, se consideraba prohibido (por el costo) el uso de un dinamómetro de rodillos para realizar las pruebas a vehículos pesados e incluso aplicar pruebas a los vehículos en uso, pero afortunadamente los recientes desarrollos en la tecnología para medir las emisiones vehiculares y consumo de combustible, ha reducido dramáticamente su costo. En este sentido, pueden apoyarse mejor los programas de reducción de emisiones y consumo de combustible, ya que pueden jugar un papel vital para los vehículos en uso, frecuentemente referidos como programas de inspección y mantenimiento (Tabla 4).

Tabla 4. Comparación de emisiones medidas en el escape y consumo de combustible en diferentes condiciones urbanas de manejo

\begin{tabular}{cccccc}
\hline \multicolumn{5}{c}{ Emisiones en $(\mathrm{g} / \mathrm{km}) \mathrm{y}$ consumo de combustible en $(\ell / 100 \mathrm{~km})$} \\
\hline & $50 \mathrm{kph}$ constante & $80 \mathrm{kph}$ constante & $\begin{array}{c}\text { Flujo } \\
\text { congestionado }\end{array}$ & Carreteras secundarias & En arterias \\
\hline $\mathrm{CO}_{2}$ & 865 & 964 & 1541 & 997 & 1080 \\
$\mathrm{NOx}$ & 8.78 & 12.16 & 13.37 & 9.89 & 11.18 \\
$\mathrm{PM}_{10}$ & 0.241 & 0.344 & 1.051 & 0.77 & 0.545 \\
C. $\mathrm{Com}$ & 32.69 & 36.43 & 58.24 & 37.47 & 40.63 \\
\hline
\end{tabular}


Los ciclos de manejo, una herramienta útil si es dinámica para evaluar el consumo de combustible ...

Existen otros ciclos para vehículos de servicio pesado, tanto en la Unión Europea como en Estados Unidos; por ejemplo, el ciclo ETC (Figura 2) desarrollado por FIGE (Forschungsinstitut Geräusche und Erschutterungen) Aachen, Alemania en 1994. En este ciclo están representadas diferentes condiciones de manejo en 3 partes, urbana, rural y autopista. La duración del ciclo es de 1800s y la duración de cada parte es de 600s. La primera parte representa el manejo en ciudad con una velocidad máxima de $50 \mathrm{kph}$, tiene frecuentes arranques, paradas y operación en vacío. La parte 2 es un modo de manejo en el área rural, iniciando con una etapa de aceleración, la velocidad promedio es alrededor de $72 \mathrm{kph}$. La parte 3 representa la forma de manejar en autopista con velocidad promedio de 88kph. FIGE desarrolló el ciclo con 2 variantes: Una como una prueba en dinamómetro de chasis y la otra para propósitos de certificación de motores, en este caso, el ciclo ETC es ejecutado en un dinamómetro para motores (Svensk Bilproving Motorcenter, 1997).

El ciclo para autobuses del condado naranja (Orange County Bus Cycle) es una prueba ejecutada en un dinamómetro de chasis que ha sido desarrollado por la Universidad del Oeste de Virgina, Estados Unidos, basado en patrones de manejo de autobuses urbanos en Los Angeles, del área de California. La velocidad del vehículo contra la duración del ciclo se ilustra en la figura 3.

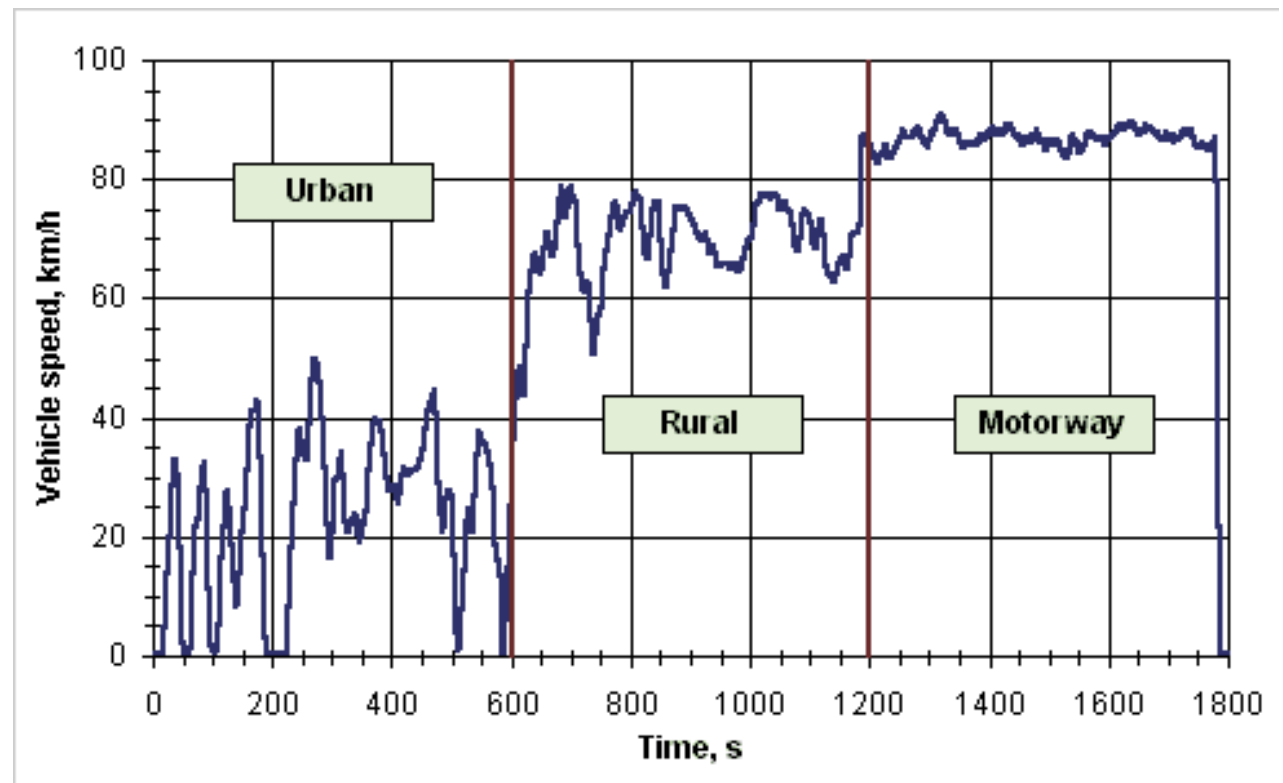

Figura 2. ETC Transient Cycle - Vehicle Speed

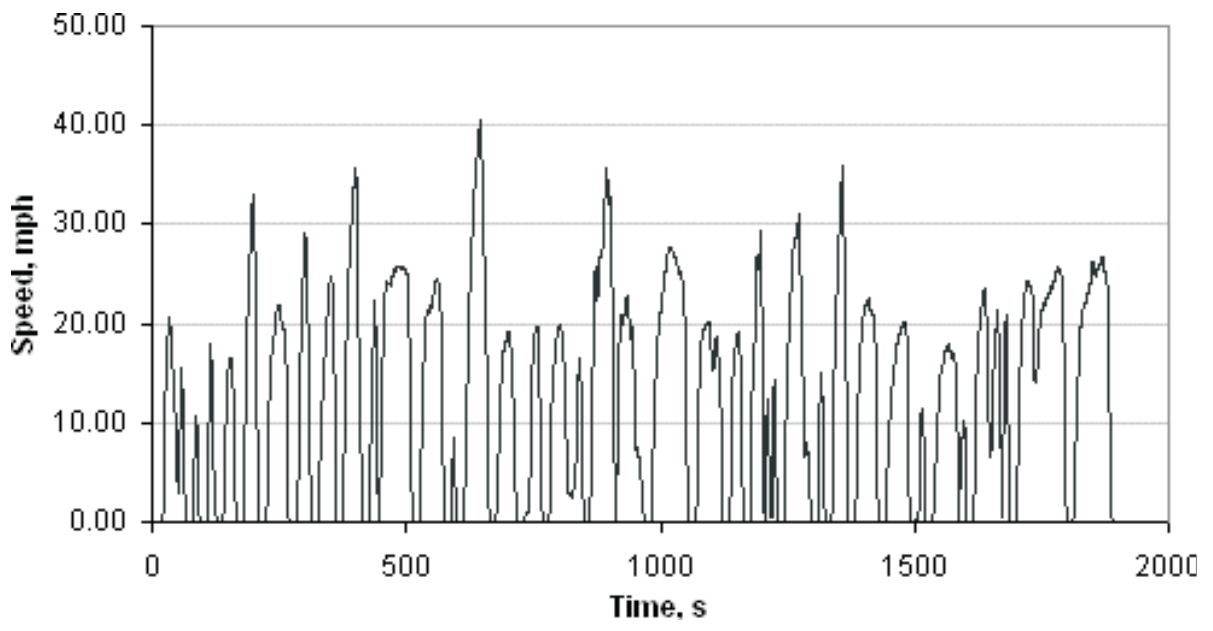

Figura 3. Orange County Bus Cycle 


\section{R. González-Oropeza}

\section{Proyecto AVERT}

El proyecto AVERT (Adaptation of Vehicle Environmental Response by Telematics) se desarrolla en Inglaterra, mismo en donde el autor de este artículo tuvo la oportunidad de participar por la Universidad de Southampton como visitante académico, con la intención de explorar algunas estrategias que puedan mejorar las condiciones del medio ambiente, a través del control de la potencia demandada a los vehículos. La idea es que el funcionamiento de los vehículos pueda ser alterado remotamente cuando se encuentren en una zona donde la calidad del aire se considera pobre, y de esta forma, mantener bajos niveles de emisiones contaminantes. Para establecer dicho control se cuenta con RTA (Road Traffic Advisor), el cual usa una Comunicación Especial de Rango Corto (DSRC, Dedicated Short Range Communication) para recibir y enviar comunicaciones entre vehículos y la estación de señales de carreteras. Las instituciones participantes son: Motor Industry Research Association (MIRA), Transportation Research Laboratory (TRL), Oxford-Brookes University (OBU) y Transportation Research Group, University of Southampton (TRG). Cada participante tiene un papel específico que desempeñar y objetivos de investigación dentro del proyecto, como se bosqueja en la figura 4.

La universidad de Southampton (TRG), usando un solo vehículo compacto, el VW Lupo V16 desarrolló
3 ciclos de manejo en el área urbana: agresivo, intermedio y pasivo. Cabe hacer notar que los fabricantes de vehículos están considerando cómo proveer a sus clientes vehículos de bajas emisiones o vehículos con cero emisiones, lo que incluye considerar vehículos con potencia motriz alternativa de la combustión interna.

\section{En México}

En lo que respecta a México, se puede hacer una breve recapitulación de cómo ha crecido el parque vehicular. En 1898, entró a México el primer automóvil, el auto era francés, marca Delaunay Belleville, hecho a mano en las fábricas de Couvier. En 1930, habían 88443 vehículos en la República Mexicana que contaba entonces con 16'588,522 habitantes, esto es, 187.6 habitantes por vehículo; en 1938, la relación era de 150, y en 1946 de 114. Posteriormente, entre las décadas de los 70's y 90 's esta relación ha oscilado entre 16 y 12 , lo que representa un aumento desmesurado del parque vehicular, en comparación con el poco tiempo de su aparición de manera comercial.

Tomando en cuenta este panorama, y además conociendo la importancia de contar con ciclos de manejo en la Ciudad de México, el grupo de trabajo del Laboratorio de Control de Emisiones de la Facultad de Ingeniería, propuso en 1998 al Gobierno del Distrito Federal un proyecto para

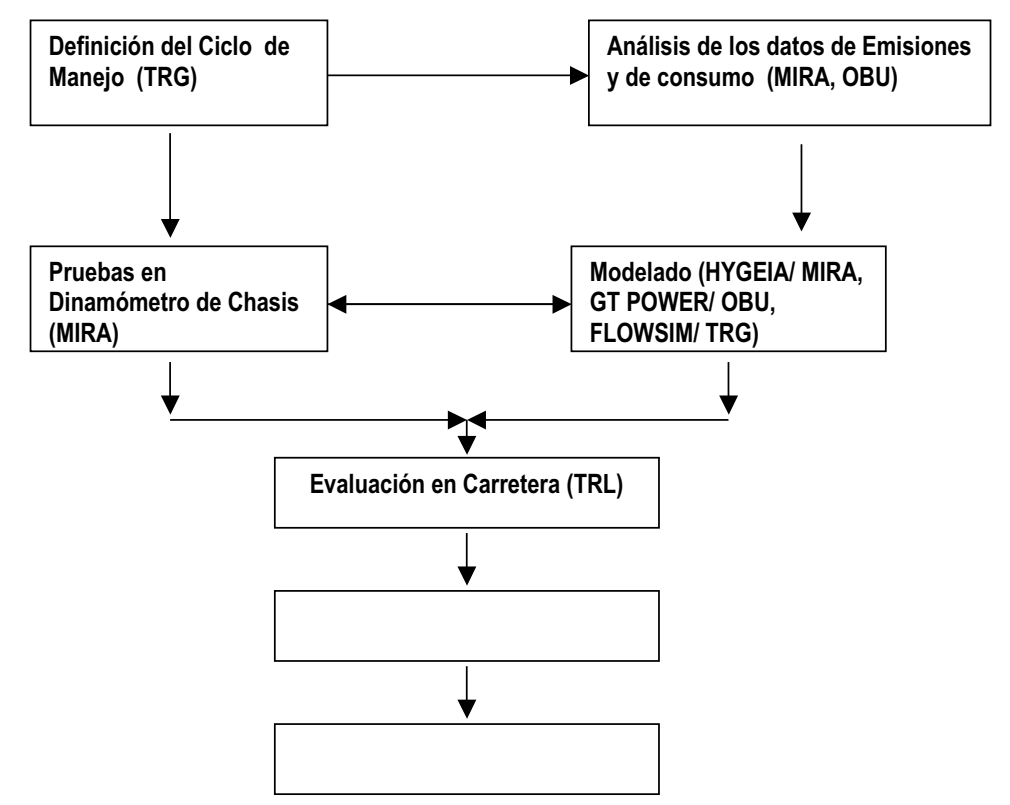

Figura 4. Organización de tareas para los integrantes del proyecto AVERT 
Los ciclos de manejo, una herramienta útil si es dinámica para evaluar el consumo de combustible ...

diseñar y construir un banco de motocicletas, así como el desarrollo de ciclos de manejo para dichas motocicletas y de vehículos ligeros. De esta manera, aprobada la propuesta, se instrumentó un vehículo Nissan, Ichi Van 1993, para monitorear segundo a segundo la velocidad del vehículo, el régimen de giro del motor, la posición de la mariposa del carburador y la temperatura del aceite de lubricación.

La recolección y almacenamiento de la información se hizo a través de un sistema de adquisición de datos a bordo (SADAB), que fue desarrollado en el mismo Laboratorio para este fin específico. El desarrollo experimental estuvo soportado sobre un modelo estadístico aleatorizado completo (González y Galván, 2003).

Los muestreos se desarrollaron entre noviembre de 1998 y mayo de 2000, abarcando 5 zonas del Valle de México como se muestra a continuación

\section{Ciclo Ciudad de México (zona Noroeste)}

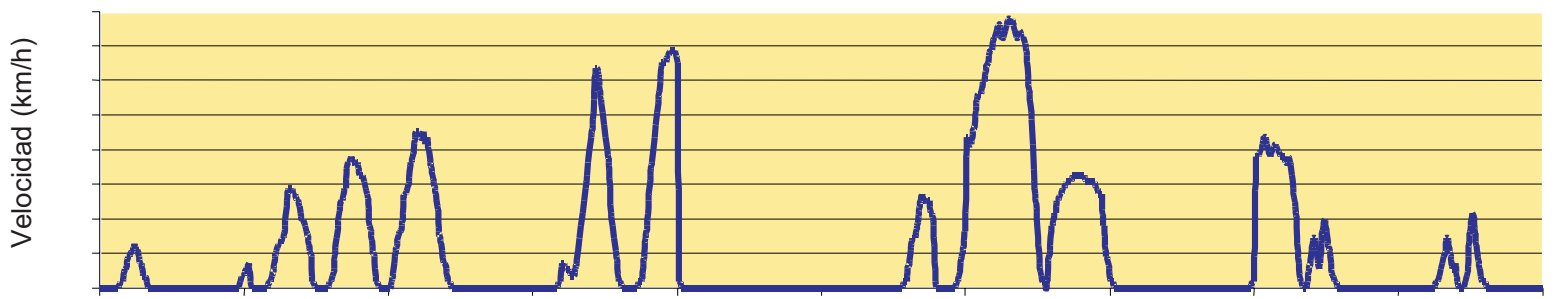

Av. Cuitláhuac y Calzada Vallejo, Egipto, Floresta, Heliopolis, Av. 5 de Mayo, Santa Lucía, Calzada de la Naranja, Calzada de las Armas, Av. de las Culturas, Eje 5 Norte, San Pablo, Av. Gasoducto, Eje 4 Norte, antigua Calzada de Guadalupe, Eje 4 Norte, Calzada Azcapotzalco la Villa, Eje 4 Norte, y Eje 1 Poniente.

Velocidad máxima $77 \mathrm{~km} / \mathrm{h}$, velocidad promedio: $18.2 \mathrm{~km} / \mathrm{h}$, duración 966 segundos, distancia recorrida:4.88 km

\section{Ciclo Ciudad de México (zona Noreste)}

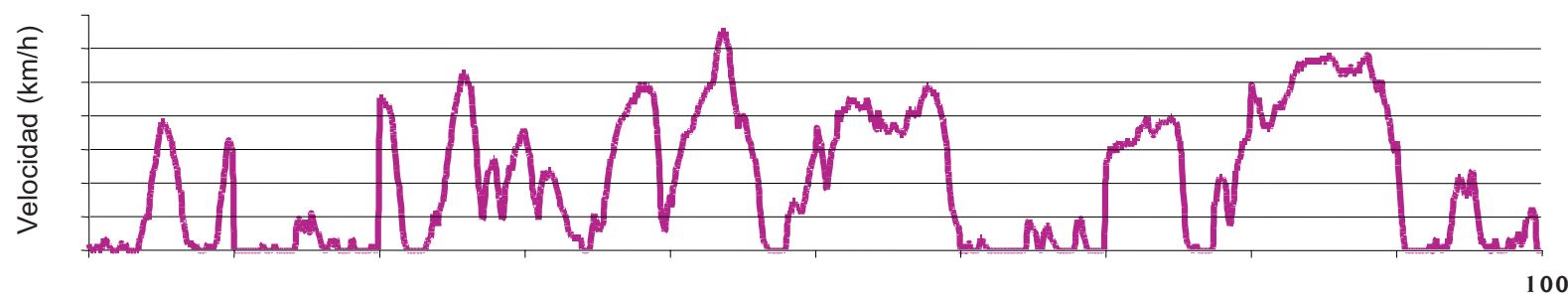

Av. de los 100 Metros, Insurgentes Norte, Othon de Mendizábal Oriente, Juan de Dios Batis, Miguel Bernal, Río de los Remedios, A. Acueducto de Guadalupe, Av. Morelos, Cerrada de Allende, Allende, Av. Morelos, Paseo de Zumarraga, Calzada de Guadalupe, Talismán, Eje 4 Norte, Av. José Loreto Fabela, Av. 608, Circuito Interior, Río Consulado, Paganini, Av. Insurgentes y Av. de los 100 Metros.

Velocidad máx: $65 \mathrm{~km} / \mathrm{h}$, velocidad promedio: $22.3 \mathrm{~km} / \mathrm{h}$, duración: $1000 \mathrm{seg}$, distancia recorrida: $6.2 \mathrm{~km}$, 


\section{Ciclo Ciudad de México (Centro)}

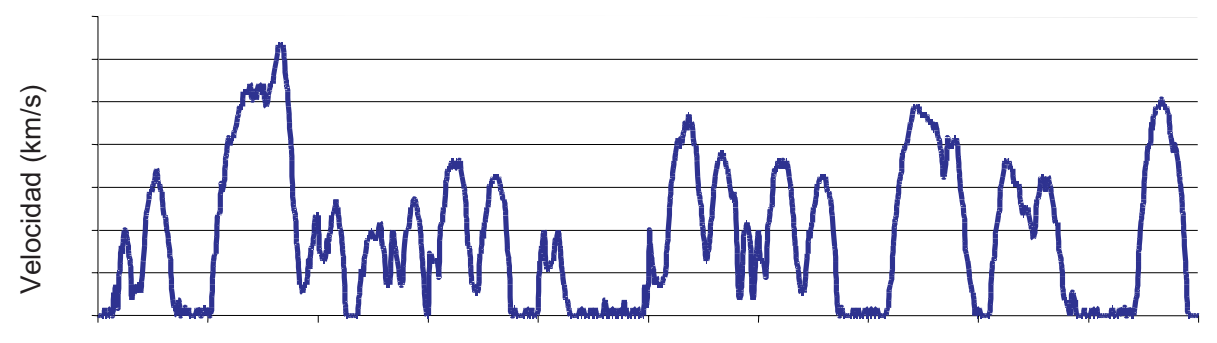

Ciclo Ciudad de México (zona Suroeste)

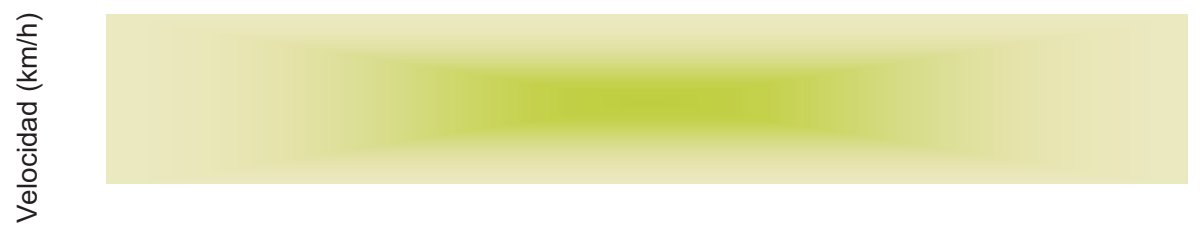

Circuito Escolar, Ingeniería, Cerro del Agua, Moctezuma, Miguel A. de Quevedo, Av. América, Pensylvania, División del Norte, Río Churubusco, Félix 
Los ciclos de manejo, una herramienta útil si es dinámica para evaluar el consumo de combustible ...

\section{Conclusiones}

1. Esta revisión no es el estado del arte en este ámbito del conocimiento; sin embargo, puede ofrecer un buen panorama de los ciclos de manejo, conocer cuáles son sus funciones y qué se está haciendo en algunos países qué desarrollan estrategias de reducción de contaminantes y consumo de combustible por parte del auto transporte.

2. Podría agregarse la medición del consumo de combustible como parte del protocolo de pruebas en la homologación de vehículos. Los fabricantes incluso, ya trabajan en ello desde hace varios años. Dicho consumo es un parámetro importante, primero porque está fuertemente vinculado con la generación de $\mathrm{CO}_{2}$ y segundo porque los combustibles más abundantes y baratos con que se cuenta hoy en día, son derivados del petróleo, que es un recurso no renovable. Tomando esto en cuenta, debe existir una legislación que limite su uso, toda vez que el sector transporte es el que presenta mayor consumo de energéticos.

3. El FTP ciertamente tiene algunas diferencias con los patrones de conducción actuales, pero pueden hacérsele ajustes que lo hagan una herramienta útil y confiable, lo cual puede provocar mejoras en los diseños de los vehículos, sin ocasionar grandes costos y disturbios en la homologación de los mismos.

4. Existen hoy en día modelos que pueden reproducir casi a la perfección patrones de manejo, valores de las emisiones y el consumo de combustible; sin embargo, siempre existe la desventaja de que los ciclos generados por dichos modelos no incluyan algunos comportamientos reales de las variables, lo cual puede causar erráticos resultados y conclusiones. Los ciclos de manejo generados, corren el riesgo de estar suavizando algunos aspectos en comparación con los datos reales del tráfico.

5. Los ciclos de manejo generados a partir de los proyectos HyZEM, ARTEMIS y MODEM, constituyen una magnífica fuente de información para establecer patrones de manejo representativos de la Unión Europea, toda vez que los muestreos se han llevado a cabo en diversos países de este continente.

6. El ciclo de manejo para autobuses, presentado por Anyon (2001) abre expectativas para considerar cambios sustanciales en las legislaciones respectivas, pero no sólo para los vehículos nuevos, sino también para aquellos que están en uso.

7. El proyecto AVERT tiene un enfoque específico en cuanto a que contemplan situaciones a corto, mediano y largo plazo para establecer estrategias que puedan ser aplicables a cualquier sistema de motorización, tomando en cuenta los 2 principales factores que se abordan en este trabajo: el consumo de combustible y los niveles de las emisiones contaminantes.

8. En cuanto a los ciclos mexicanos, se han desarrollando siguiendo una metodología propia, y los datos obtenidos coinciden plenamente con los rangos mostrados en la literatura consultada. El sistema de adquisición de datos fue desarrollado por alumnos que elaboraron su tesis en el LCE y significa un potencial que pueda explotarse en beneficio de la capacitación de los propios estudiantes, así como de los ámbitos donde se requiera este tipo de equipo de muestreo. Los ciclos de manejo obtenidos, han tenido buena aceptación en algunas de las industrias automotrices del país y en algunas dependencias gubernamentales. Se han utilizado para evaluar dispositivos y aditivos en relación con las emisiones contaminantes y el consumo de combustible, y como un ejemplo de su utilidad, comentaremos que con base en ellos se ha evaluado el desempeño del vehículo híbrido Prius de Toyota en el LCE.

\section{Referencias}

EPA (1998). Federal Test Procedure Review Project: Preliminary Technical Report. May 1993, March, pp. 1-207.

André M. Driving Cycles Development: Characterisation of the Methods. SAE Technical Paper Series 961112.

Per Kågenson. (1998). Cycle-Beating and the EU Test Cycle for Cars. European Federation for Transport and Environment, TEE 98/3 November. 
Robert M.M., Van Den Brink, Bert Van Wee. (2001). Why a Has Car-Fleet Specific Fuel Consumption not Shown any Decrease Since 1990? Quantitative Analysis of Dutch Passenger Car-Fleet Specific Fuel Consumption. Transportation Research, Part D6, pp. 75-93.

Lee S. y Wienke T. (1994). New Car test and Actual Fuel Economy; yet Another Gap? Transport Policy, 1 (4), pp. 257-265.

Anyon P. (2001). Green Buses on Schedule. Smart Urban Transport, Vol. 1, Sept. pp. 14-18.

Esteves-Boot A., Muneer T., Kirby H., Kubie I., Hunter J. (2001). The Measurement of Vehicular Driving Cycle Within the City of Edinburgh. Transportation Research, Part D 6, pp. 209-220.

Michel A. (2001). Driving Cycles Deriven from Real-World in-Vehicle Measurement for Passenger Cars and Light Duty Vehicles: Principles, Database and Main Results-Particular case of the ARTEMIS Driving Cycles. INRETS France.

Ericsson E. Variability in exhaust emission and fuel consumption in urban driving. Lund University, Sweden.

Svensk Bilproving Motorcenter (1997). Report 9707.

González-Oropeza R. y Galván-Zacarías A. (2003). Desarrollo de ciclos de manejo característicos de la Ciudad de México. Memorias del IX Congreso Anual Investigación y Desarrollo Tecnológico en Ingeniería Mecánica, pp. 535-544, SOMIM, México.

Secretaría del Medio Ambiente y Recursos Naturales (SEMARNAT). Ley general del equilibrio ecológico y la protección al ambiente articulo $3^{\circ}$ fración XIV.

\section{Bibliografía sugerida}

Brackstone M., McDonald M. y Sultan B. (1689). Dynamic Behavioral Data Collections Using an Instrumented Vehicle. Transportation Research Record, paper No. 99-2535.

De Vliegers I. (1997). On-board Emission and Fuel Consumption Measurement Campaign on Petrol- Driven Passenger Cars. Atmospheric Environment, Vol. 31, No 22, pp. 3753-3761.

Directive 1999/96/EC of December 13, 1999.

EPA, 40 CFR Parts, 80, 85, 86.

Ericsson E. (2001). Independent Driving Pattern Factors and their Influence on Fuel-Use And Exhaust Emission Factor. Transportation Research, part D 6, pp. 325-345.
Felstead T., Marsden G., McDonald M.(2001). AVERT-Developing Drive Cycle to Illustrate Driving Conditions in Southampton. Transportation Research Group, University of Southampton, UK. A Interim Report.

Hackney J. y Neufville R. (2001). Life Cycle Model of Alternative Fuel Vehicles: Emissions, Energy, and Cost Trade-Off. Transportation Research, Part A 35, pp. 243-266.

Michel A. (1998). Building-Up of Representative Driving Cycle for the Measurement of Pollutant Emissions from Road Vehicles. Doctoral thesis, INRETS.

Nesvitt K., Sperling D. (2001). Fleet Purchase Behaviour: Decision Process and Implications for new Vehicle Technologies and Fuels. Transportation Research. Part C 9, pp. 297-318.

Powell M. (2000). A Model to Represent Motorcycle Behaviour at Signalised Intersections Incorporating and Amended First Order Macroscopic Approach. Transportation Research, Part A 34, pp. 497-514.

Varhelyi A., Mäkinen T. (2001). The Effects of in-car Speed Limiters: Field Studies. Transportation Research, Part C 9, pp. 191-211.

Vlieger I. De, Keurebere D. De, Kretzschmar J.G. (2000). Environmental Effects of Driving Behaviour and Congestion Related to Passenger Cars. Atmospheric Environment, 34, pp. 4649-4655. 
Los ciclos de manejo, una herramienta útil si es dinámica para evaluar el consumo de combustible ...

\section{Semblanza del autor}

Rogelio González-Oropeza. Estudió la licenciatura y la maestría en ingeniería mecánica en la Facultad de Ingeniería de la UNAM. Finalizó el doctorado en la Universidad Politécnica de Valencia, España. Es profesor de la Facultad de Ingeniería y labora en el Departamento de Termoenergía y Mejoramiento Ambiental. Ha recibido diversas distinciones como: uno de los 3 primeros lugares en la maestría de ingeniería mecánica, la calificación Cum Laude en la tesis doctoral, la Cátedra Especial en 2 ocasiones y como revisor técnico de la Sociedad Mexicana de Ingeniería Mecánica. Ha sido coordinador y expositor en 12 cursos de especialización y diplomados en el área de los motores de combustión interna alternativos, termodinámica y didáctica de la física, ha dirigido tesis de licenciatura, maestría y doctorado y ha sido asesor de la Comisión Nacional para el Ahorro de Energía. Asimismo, ha colaborado con grupos de trabajo de su área en la Universidad Politécnica de Valencia, España y en la University of Southampton, England. Actualmente es miembro de la Comisión Evaluadora del PRIDE del área de las Ciencias Físico Matemáticas y de las Ingenierías de la Facultad de Estudios Profesionales, Cuautitlán UNAM, y miembro del Comité Consultivo Nacional de Normalización para Protección Ambiental, que preside la Secretaría del Medio Ambiente y Recursos Naturales. 